\title{
Elk Populations in the Western United States and Canadian Provinces
}

\section{Discover how game agencies count elk, why, and the accuracy of the estimates.}

\section{By Lance T. Vermeire, Mark C. Wallace, and Robert B. Mitchell}

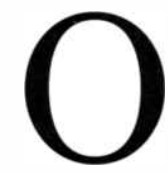

ne of the ways wildlife biologists determine elk population management strategies and evaluate the success of previous management is to monitor population size. This can be a difficult task with animals that know few boundaries. However, demands for improved elk population estimates are increasing throughout much of the western United States and Canada.

Part of the interest comes from the desire to reintroduce elk to their former range and increase existing populations. Conservation efforts have been generally successful. The Rocky Mountain Elk Foundation reported more than 782,000 elk in North America in 1989 and 968,000 elk a decade later. The number of elk is small relative to presettlement figures or the current number of livestock. However, the distribution of elk is quite reduced, and local populations are often dense. So, there is some concern about the effects locally large and growing herds may have on rangelands.

Accurate population estimates may be necessary to determine appropriate stocking rates for rangeland shared by elk and domestic livestock. The top 4 elk states have 1 elk for every 14 head of cattle. The potentially delicate balance of maintaining desired elk populations and healthy rangelands may hinge on the accuracy of these estimates. We contacted state and provincial wildlife agencies in the United States and Canada to determine the procedures used to assess elk populations, how the estimates were used, and the level of accuracy the agencies believed those procedures provided.

\section{Background on Estimation Techniques}

Different estimation techniques have been developed for specific population conditions, and decision support systems have been suggested for choosing the most appropriate method. Method selection should be based on the conditions of the population being examined and the information needed from the estimate. Qualitative indices, such as hunter surveys, may suffice when only relative density estimates (trends) are needed. However, more precise, quantitative density estimates require direct measures. These may include complete counts, incomplete counts, capture techniques, or modeling based on results of these methods.

Complete counts assume all animals are observed, usually driving them out of an area or using aerial photographs of animals in open landscapes. The feasibility of obtaining such a count naturally decreases with larger sample areas and increasing visual obstructions, such as trees. Incomplete counts do not assume all animals are observed, so an estimate of the fraction observed is used to adjust counts. Capture techniques utilize the ratio of captured or marked animals to noncaptured or nonmarked animals over time to derive estimates. Capture methods are akin to shepherds using a black sheep for a given number of white sheep. The shepherd can make quick surveys based on the assumption that all sheep are present if all black sheep are observed.

Within these general classes, techniques can be further refined to match the conditions of the population. Information on individual animals is best obtained with mark-recapture methods. If such in- 


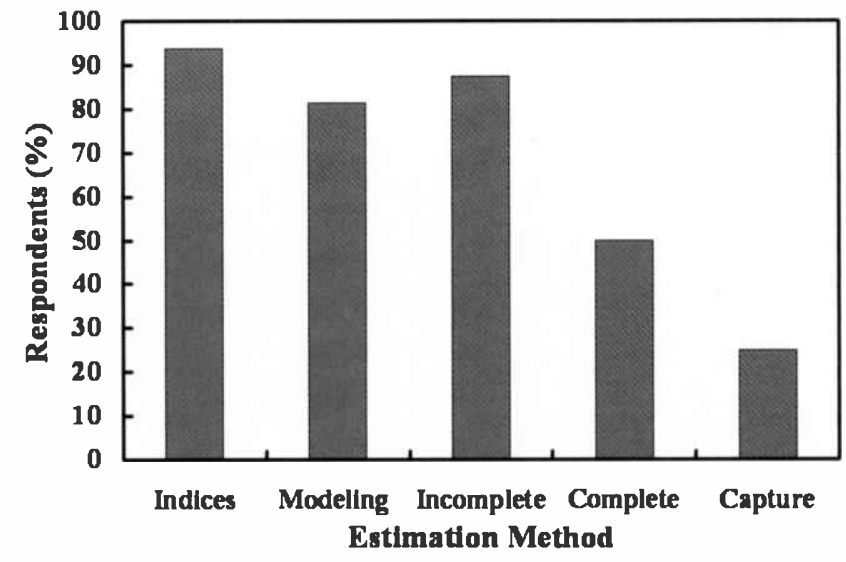

Figure 1. Number of state and provincial wildlife agencies reporting the use of indices, modeling, incomplete counts, complete counts, and capture methods to estimate elk population size in 1997-1998.

formation is not required, the mobility, exploitation, and distribution of the animals must be considered. Counting animals in blocks or large quadrats is suggested for animals that are immobile relative to the sampling method. Block counts are also recommended for high-density populations that are exploited or nonrandomly distributed. Line transect, distance, and variable plot methods apply to animals that may move during sampling. Animal movement during sampling increases the risk that some animals will not be counted and some will be counted multiple times. Mobile populations currently experiencing selective exploitation can be estimated by change-inratio methods. For example, male-to-female ratios may be monitored before and after hunting season when males are selectively hunted.

\section{Survey of Game Agencies}

We designed an 18-question survey to determine 1) which elk population estimation techniques were used by wildlife agencies, 2) how the estimates were being used, and 3) the perceived level of accuracy desired and achieved with those methods. Seventeen wildlife agencies were identified from western United States and Canadian states and provinces with estimated elk populations greater than 1,000. Agencies were contacted by phone to explain the survey and determine their willingness to participate. Four weeks after surveys were mailed, agencies that had not responded were contacted again by phone and offered assistance with completing the survey. A second copy of the survey was mailed to nonrespondents. Following initial analysis, agencies were contacted by phone and asked 18 follow-up questions to clarify and expand on initial reports. Completed surveys were received from 16 of the 17 agencies, representing $98 \%$ of the 1998 elk population in the United States and Canada.

\section{How Game Agencies Estimate Elk Populations}

Most agencies used some combination of indices, population models, incomplete counts, or complete counts (Fig. 1). All but 1 agency used indices to assess population trends. Hunter success was the most common index used. The POP II (Version 7, 1995, Fossil Creek Software, Fort Collins, CO) and modified versions of the program were the most common modeling tools used, but age/sex/kill models were frequently applied as well. At least 5 states and provinces using incomplete counts were applying or experimenting with visibility models.

Table 1. Season and frequency of elk population surveys conducted by state and provincial wildlife agencies. Sums may exceed the number of respondents when season of sampling varies within agency or when an agency samples during multiple seasons within a year.

\begin{tabular}{lccccc}
\hline \hline & & \multicolumn{4}{c}{ Timing of survey } \\
\cline { 3 - 5 } Surveys/year & Responses & Summer & Autumn & Winter & Spring \\
\hline$<1$ & 7 & 1 & 1 & 7 & 2 \\
1 & 3 & 0 & 0 & 3 & 1 \\
& 6 & 2 & 3 & 4 & 1 \\
\hline
\end{tabular}


Visibility models adjust estimates based on the percentage of animals missed in various habitat types and with different weather and topographic conditions. Half the agencies reported using complete counts. However, use of the term "complete count" appears to have included counts on sample plots or specific herds that were extrapolated to represent a larger area or the greater herd. Complete counts on sample plots still require the assumption that no elk go unobserved in surveys.

Nine of the 16 agencies conducted 1 or more counts per year (Table 1). Counts were typically made in winter because elk tend to congregate at lower elevations during winter. Snow cover also tends to enhance the ability of observers to detect the animals. Spring counts were limited predominantly to March and may be considered an extension of winter counts. Summer counts were used to determine age or sex ratios. Autumn was generally selected for prehunt counts that were later associated with posthunt surveys.

Surveys were most commonly conducted with helicopters and multiple observers. However, there was wide use of fixed-wing aircraft, ground counts, and single observers as well. The combination of methods used varied not only among states and provinces but often within the same state or province. Three factors explained the use of multiple methods within an agency. First, the relative effectiveness of methods often changes with location or landscape. Second, broad application of the preferred method may not be feasible with budgetary, labor, or time constraints. Finally, some agencies were testing alternative methods to improve estimates.

\section{Why Estimates Are Obtained}

The highest-ranking reason for estimating population size was to determine the level of harvest needed to meet management goals (Fig. 2). Additional reasons, in decreasing order of ranked importance, were to evaluate herd health, effectiveness of habitat management, and management effects on crop depredation. We encouraged agencies to add other reasons if they were not included in our selection. Two agencies noted that political pressure was the second most important reason for surveying elk populations, and 1 reported that monitoring effects

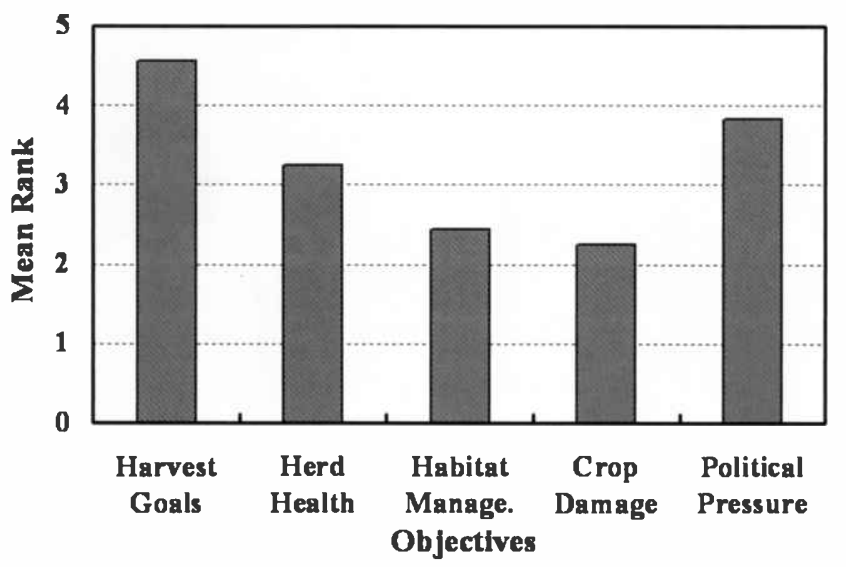

Figure 2. Reported reasons for determining elk population size as ranked by state and provincial wildlife agencies. Rank of 5 represents maximum importance. Political pressure was ranked on the same scale but at a later date and separately from other categories.

of human developments was their third most important reason.

On the follow-up survey, 10 of 14 responding agencies reported that political pressure was driving their agency's need for population estimates. Political pressure received a mean rank of 3.8 on a scale of 5, making it the second most important reason agencies needed population estimates (Fig. 2). The agricultural sector and hunters were the dominant lobbying groups. Agriculture generally claimed there were too many elk, and hunters claimed there were too few.

\section{Estimate Accuracy, Perceived and Achieved}

Sampling intensity among agencies was highly variable. On average, a maximum of about $60 \%$ of

Table 2. Desired and current elk population estimate error rates and standard errors by human and elk population category for western state and provincial wildlife agencies. Standard errors are reported in parentheses.

\begin{tabular}{|c|c|c|c|}
\hline \multicolumn{2}{|c|}{ Relative population } & \multicolumn{2}{|c|}{ Estimate error $( \pm \%)$} \\
\hline Human & Elk & Desired & Current \\
\hline Low & Low & $22(6)$ & $18(4)$ \\
\hline Low & High & $22(6)$ & $33(6)$ \\
\hline High & Low & $13(3)$ & $16(4)$ \\
\hline High & High & $11(3)$ & $26(5)$ \\
\hline
\end{tabular}




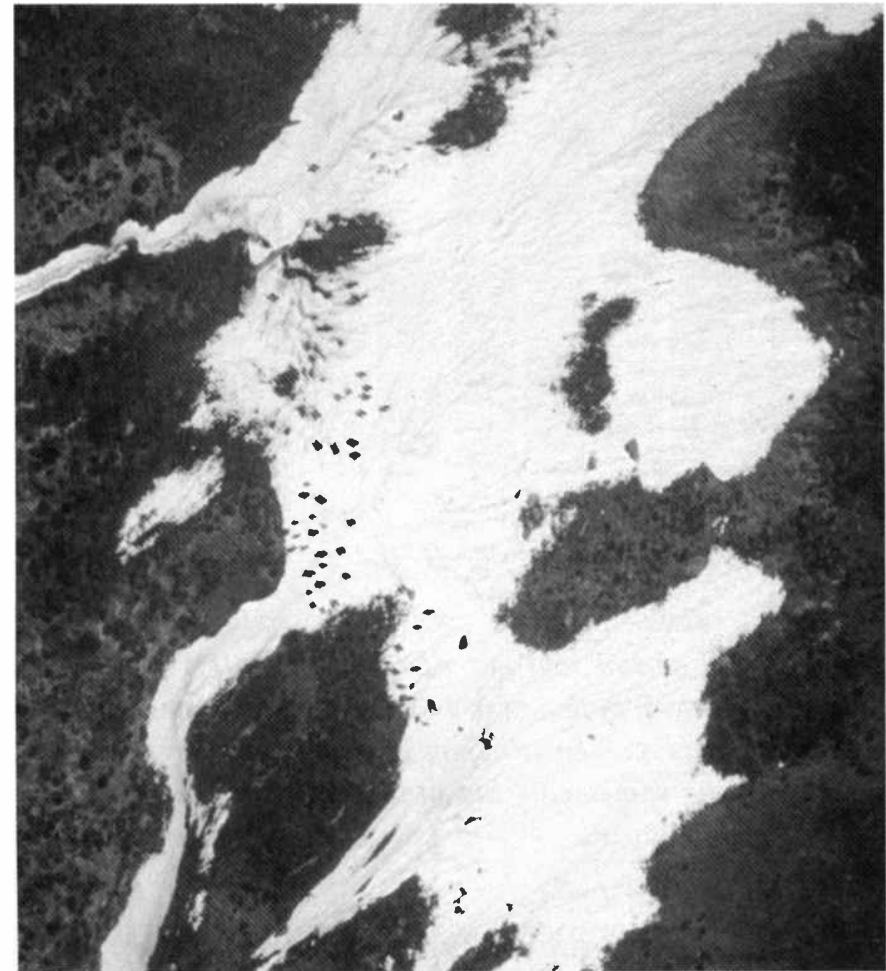

Aerial counting of elk on snow fields. The black dots represent individual elk. Elk are not visible in the dark areas.

elk populations and ranges were reported to be sampled. Two agencies did not report the level of accuracy they believed was needed to provide an acceptable estimate. On average, the agencies that reported desired accuracy concluded they would like estimate errors reduced to within $17 \%$ of the true population.

One of the 16 respondents did not venture to guess the level of accuracy their methods achieved.

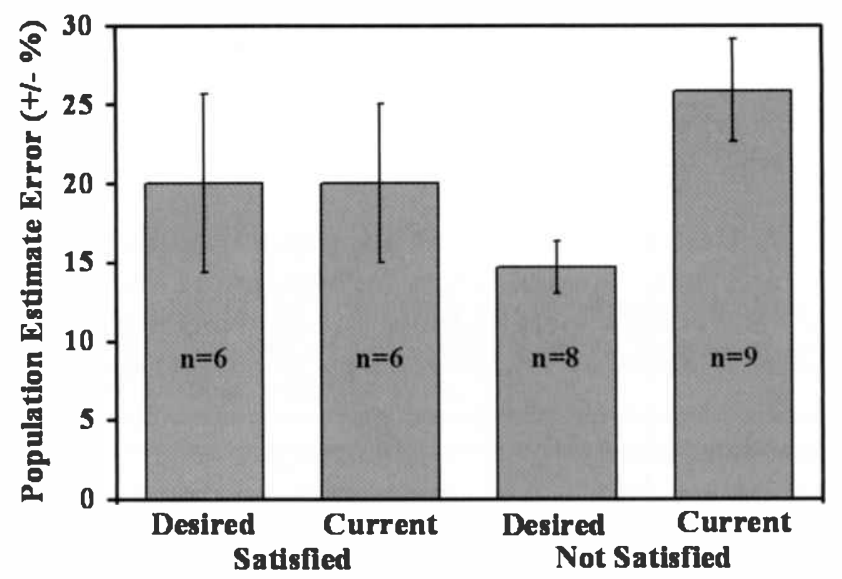

Figure 3. Desired and current error rates of elk population estimates by state and provincial wildlife agencies reporting satisfaction or dissatisfaction with current result.
Error rates for current methods were reported to be equal to or better than desired levels for 8 of the 15 agencies that responded. However, when asked directly whether agencies were satisfied with their current accuracy levels, only 6 reported satisfaction. One of those 6 agencies reported current error was greater than that desired.

Agencies not satisfied with current estimates reported a smaller desired error and greater current error than those reporting current satisfaction (Fig. 3 ). Four agencies said their management needs could be sufficiently met with trend data, but none of these agencies was among those reporting satisfaction with current accuracy levels. The discrepancy between meeting management needs and being satisfied with trend data indicates greater accuracy was being demanded (internally or externally) than was necessary to effectively manage elk.

\section{Sorting It Out}

We believe political pressure and the size of elk herds are primary factors controlling why and how wildlife agencies are estimating populations. Data were partitioned into 4 groups, based on the population size of humans and elk. States and provinces with small elk populations reported greater accuracy than those with large elk populations (Table 2). The small populations are generally not less dense, just limited in distribution. The concentration of elk in smaller areas allows better coverage of the population. So, accuracy of estimates will be greater or less survey effort will be required.

States with sparse human populations have a lower probability of conflict between elk and human uses. Therefore, their counts are generally considered complete, and their acceptable error rates are greater than those of agencies in more densely populated areas (Table 2). For example, a Rocky Mountain state with one of the largest elk populations but a sparse human population based its estimates solely on hunter surveys.

However, states and provinces with substantial conflict between elk and human use (eg, the Pacific Northwest) feel pressure for improved estimates to effectively manage potential conflicts. Many of these states and provinces are applying visibility models, probably because there is currently a lot of faith placed in such models. One northwestern state 
derives its estimates using a visibility model that it classified (incorrectly) as a complete count on our survey. Some states in the Southwest are starting to feel some of the same pressures and are adapting visibility models as well.

Colorado is a prime example of an agency being driven by political pressure. The agency is required to pay for damages caused by elk. In response, they are vigorously testing estimation methods and intensively sampling, conducting up to 4 surveys per year. Colorado was one of the few agencies that reported the level of accuracy they achieved was less than that desired. Their acceptable level of error was also greater than the average of all others. Colorado is actively testing survey methods. So, their stated error rates probably better reflect the strength of their assessments rather than a weakness in their results relative to other agencies.

When asked what agencies would do differently if cost were not an issue, the overwhelming response was more sampling over time and space rather than use of a different method. In this respect, complications in the estimation of elk populations may be analogous to those of vegetation monitoring. The greatest limitation to accurate estimates may not be a matter of methodology. Rather, the difficulties are in the selection of a method that will be accurate with the financial and human resources available to derive estimates over large areas in a short period.

Authors are rangeland ecologist, USDA-ARS, Fort Keogh Livestock and Range Research Laboratory, 243 Fort Keogh Road, Miles City, MT 59301; associate professor, Department of Range, Wildlife, and Fisheries Management, Texas Tech University, Lubbock, TX 79409-2125; and rangeland scientist, USDA-ARS, Wheat, Sorghum, and Forage Research Unit, $344 \mathrm{Keim} \mathrm{Hall,} \mathrm{E.C.,}$ University of Nebraska, Lincoln, NE 68583. Vermeire and Mitchell were formerly research assistant and associate professor, Department of Range, Wildlife, and Fisheries Management, Texas Tech University. The authors appreciate the individuals and wildlife agencies from the following states and provinces for their participation in the survey: Alaska, Alberta, Arizona, British Columbia, California, Colorado, Idaho, Montana, Nevada, New Mexico, Oregon, Saskatchewan, South Dakota, Utah, Washington, and Wyoming. USDA Agricultural Research Service, Northern Plains Area, is an equal opportunity/affirmative action employer, and all agency services are available without discrimination. 